\title{
Tick-Borne Encephalitis Virus
}

National Cancer Institute

\section{Source}

National Cancer Institute. Tick-Borne Encephalitis Virus. NCI Thesaurus. Code C112422.

A species of enveloped, spherical and icosahedral viruses in the Flaviviridae family and Flavivirus genus. The genome is composed of non-segmented, single-stranded, positivesense RNA. Tick-borne encephalitis viruses are found in ticks of the Ixodes species and humans and cause tick-borne encephalitis in humans. Tick-borne encephalitis viruses are transmitted via the bite of infected ticks, primarily Ixodes ricinus or Ixodes persulcatus. 